\title{
Cross-Evaluation of Entity Linking and Disambiguation Systems for Clinical Text Annotation
}

\author{
Camilo Thorne \\ Universität Mannheim \\ camilo@informatik.uni- \\ mannheim.de
}

\author{
Stefano Faralli \\ Universität Mannheim \\ stefano@informatik.uni- \\ mannheim.de
}

\author{
Heiner Stuckenschmidt \\ Universität Mannheim \\ heiner@informatik.uni- \\ mannheim.de
}

\begin{abstract}
In this paper we study whether state-of-the-art techniques for multidomain and multilingual entity linking can be ported to the clinical domain. To do so, we compare two known entity linking systems, BabelFly and TagMe, that leverage on Wikipedia and DBpedia, with the standard clinical semantic annotation and disambiguation system, MetaMap, over the SemRep clinical word sense disambiguation gold standard. We show that BabelFly and especially TagMe, while achieving decent precision on clinical annotation, outmatch MetaMap's F1-score.
\end{abstract}

\section{CCS Concepts}

-Information systems $\rightarrow$ Information extraction; •Computing methodologies $\rightarrow$ Lexical semantics; Ontology engineering;

\section{Keywords}

Entity linking; semantic annotation; DBpedia; clinical NLP.

\section{INTRODUCTION}

Nowadays, textual information increasingly pervades healthcare. From electronic health records, to clinical papers, online dictionaries or clinical guidelines, an ever larger share of knowledge underpinning clinical decision-making is available as raw, unstructured text [5]. This has motivated the application and adaptation of natural language processing (NLP) techniques to index and search, but also to extract knowledge from clinical text.

An important task in information extraction is semantic annotation in the form of entity extraction ${ }^{1}$. To solve this task we need to perform two steps: (1) identify entities (nouns, noun phrases) within an text; (2) identify or resolve the meaning of such (generic) entities within such text by linking them to a sense repository. Consider the following example:

Low dose pramipexole is neuroprotective in the MPTP mouse model of Parkinson's disease.

\footnotetext{
1 We assume following Hobbs in [6] a broader notion of entity that encompasses not only names, but any noun or noun phrase in a sentence.

Permission to make digital or hard copies of all or part of this work for personal or classroom use is granted without fee provided that copies are not made or distributed for profit or commercial advantage and that copies bear this notice and the full citation on the first page. Copyrights for components of this work owned by others than ACM must be honored. Abstracting with credit is permitted. To copy otherwise, or republish, to post on servers or to redistribute to lists, requires prior specific permission and/or a fee. Request permissions from permissions@ acm.org. SEMANTiCS 2016, September 12-15, 2016, Leipzig, Germany

(C) 2016 ACM. ISBN 978-1-4503-4752-5/16/09. . $\$ 15.00$

DOI: http://dx.doi.org/10.1145/2993318.2993345
}

In (*) three generic entities have been identified, two clinical (bold) and one related to world knowledge. By "Pramipexole" is meant a kind of drug that activates dopamine receptors; by "model" is meant a theoretical framework; and by "Parkinson's disease" the known neurodegenarative disease.

To solve (1) and (2) we need to solve an entity linking (EL) and a word-sense disambiguation (WSD) task: to identify the senses $\operatorname{sen}(e)$ of a (generic) entity $e$ within a sense inventory and then determine, based on its sentential context, which is the one effectively used. While there are systems and sense inventories that can extract and disambiguate either clinical or world-knowledge entities, the question arises as to whether there are systems capable of annotating both.

For the clinical domain, the standard resource is the US national Library of Medicine (NLM) Unified Medical Language System (UMLS) Metathesaurus ${ }^{2}$. For world (encyclopedic) knowledge known resources are, e.g., WordNet [3]. However, more recently, BabelNet [13] has arisen as a multilingual thesaurus that incorporates lexical knowledge extracted from Wikipedia. Moreover, linked open data, in the form of open domain knowledge bases or ontologies such as DBpedia [2], also based on encyclopedic knowledge extracted from Wikipedia, have also been proposed as sense inventories [8] for EL and WSD. So has been Wikipedia itself [7]. It is known that Wikipedia covers a significant portion of clinical senses [14]. Thus, Wikipedia-driven annotators appear like good candidates for annotating both clinical and non-clinical entities, but their performance in the clinical domain remains relatively unknown.

The contributions of this paper are three: (1) We provide a comparative evaluation of clinical annotation resources (MetaMap) and two state-of-the-art Wikipedia-driven linking systems, BabelFly and TagMe, over a known clinical gold WSD corpus, the SemRep corpus. (2) We exploit open linked data to align all senses (gold senses and annotator senses) to DBpedia. (3) We study whether the annotations returned by each annotator are semantically similar among themselves and with respect to the gold corpus.

\section{SENSE REPOSITORIES}

UMLS. The UMLS Metathesaurus is the main thesaurus used for the clinical domain. Developed since the early 90's by the $\mathrm{NLM}^{3}$, it is built around the notion of a clinical concept, to which a so-called concept unique identifier (CUI) is associated. Concepts are organized into semantic types, and related by a certain number semantic relations, giving rise to a so-called semantic network.

WordNet. WordNet, developed in the 90s at Princeton, is a wellknown sense inventory for standard English. It is organized around

\footnotetext{
${ }^{2}$ https://www.nlm.nih.gov/research/umls/

${ }^{3}$ https://uts.nlm.nih.gov
} 


\begin{tabular}{r|l|l|l} 
& sense & sense ID & DBpedia URI \\
\hline \hline Clinical & pramipexol & $\mathrm{C} 0074710$ & $\begin{array}{l}\text { http://dbpedia.org/resource/Pramipexole } \\
\text { http://dbpedia.org/resource/Parkinson_disease }\end{array}$ \\
(Gold) & Parkinson disease & $\mathrm{C} 0030567$ & $\begin{array}{l}\text { http://dbpedia.org/resource/Pramipexole } \\
\text { http://dbpedia.org/resource/Parkinson_disease }\end{array}$ \\
\hline \hline MetaMap & pramipexol & $\mathrm{C} 0074710$ & http://dbpedia.org/resource/Pramipexole \\
& Parkinson disease & $\mathrm{C} 0030567$ & http://dbpedia.org/resource/Pramipexole \\
\hline BabelFly & ATC_code_N04BC05 & bn:03124207n & https://en.wikipedia.org/wiki/Pramipexole \\
\hline TagMe & pramipexole & https://en.wikipedia.org/wiki/Parkinson's_disease & http://dbpedia.org/resource/Parkinson's_disease
\end{tabular}

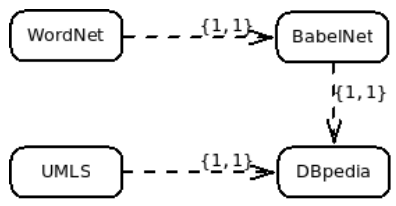

Figure 1: Left: Semantic unresolved annotations for example (*). On the first two rows, gold standard clinical senses, together with their UMLS CUI and the corresponding DBpedia URIs. Next, corresponding MetaMap, BabelFly and TagMe annotations. MetaMap senses are UMLS CUIs. BabelFly senses, BabelNet synset IDs. The WordNet baseline returned no links for either (generic) entity. Right: Outline of the mappings among the resources. While only partial they are (1) one-to-one and (2) pivot around DBpedia.

the notion of word sense, viz., a context that uniquely characterizes a particular use of a given word or term. Senses are organized into synsets, a class of synonymous senses, among which hold a number of so-called lexical relations, such as, synonymy, antonymy, hypernonymy, homonymy, etc. Senses and synsets are defined via glosses. Currently, WordNet ${ }^{4}$ comprises 117,000 synsets.

Wikipedia. Wikipedia ${ }^{5}$ is an online multilingual, cross-domain encyclopedia covering around 292 languages (282 active) and currently 5,145,674 articles for English alone. It is loosely structured around a taxonomy of topics. Wikipedia has been used a resource for (generic) entity recognition, linking and extraction as a large number of its articles define or describe entities.

DBpedia. DBpedia [2] on the other hand, emerged in the 2000s partly as an attempt to add further structure to Wikipedia, via semantic technologies (the Resource Description Framework or RDF data model) and open information extraction techniques. DBpedia is built around resources, encoding Wikipedia entries, each identified by a unique resource identifier (URI). Resources are organized into a knowledge base, viz., into triples describing resource typing and binary relations among resources, but also intensional knowledge over such types and relations. DBpedia covers 111 languages, and 65,143,840 extensional triples for English alone ${ }^{6}$.

BabelNet. BabelNet [13] was built by automatically extending WordNet with lexical knowledge gathered from Wikipedia, from several multilingual extensions of WordNet and WordNets developed for languages other than English. As such, it extends WordNet via the notion of a multilingual babelsynset, which, in addition to multilinguality, contains links to DBpedia resources in the form of DBpedia resource URIs. Currently, BabelNet ${ }^{7}$ covers around 14 million babelsynsets, and 271 languages.

\section{ANNOTATION EVALUATION}

Sense Linking. Two semantic resources $T$ and $T^{\prime}$, are said to align when there exists a mapping $f: T \rightarrow T^{\prime}$ that assigns to each sense $s \in T$ a unique sense $f(s) \in T^{\prime}$. Such mappings, when they exist, tend to be partial at best. They are however critical for evaluating results among annotators that rely on different semantic resources, in particular when they give rise to a "pivot" resource into which they can be all mapped. It is known that world-knowledge resources can be mapped in such manner to DBpedia. Interestingly, this is also possible, albeit in a more restricted form, for the UMLS.

BabelNet strictly extends WordNet [13], giving rise to an injective, total mapping of WordNet synsets to BabelNet babelsynsets. Babelsynsets in their turn can be partially mapped into DBpedia, and assigned a DBpedia resource URI. Finally, DBpedia contains a resource URI for each Wikipedia entry [2].

\footnotetext{
${ }^{4}$ https://wordnet.princeton.edu

5 http://wikipedia.com

${ }^{6}$ http://dbpedia.org ${ }^{7}$ http://babelnet.org
}

Regarding UMLS CUIs, the mapping is less obvious, but still possible. In [11] it has been shown that UMLS CUIs can be partially aligned with DBpedia URIs via Medical Subject Headings $(\mathrm{MeSH})$ terms. MeSH is a controlled vocabulary of subject headings and related glosses (short descriptions or definitions). Most UMLS CUIs can be associated to a unique MeSH term, which in its turn, can be linked or mapped to a DBpedia URI. This mapping has given rise to a linked open data knowledge base, Liked Life $\operatorname{Data}^{8}$ that can be used to partially align the UMLS with WordNet, BabelNet and DBpedia. Figure 1, right, shows the relationships among the different resources.

\subsection{Annotation and Linking Systems}

WordNet (baseline). As customary for systems that rely on WSD, we implemented as baseline a WordNet-based annotator using the known Lesk WSD algorithm [10]. The Lesk algorithm maximizes the bag-of-words similarity measure between entity $e$ 's sentence $S$ (represented as a bag of words) and the glosses of its candidate senses (WordNet synsets) $s \in \operatorname{sen}(e)$.

MetaMap. MetaMap [1] is a state-of-the-art clinical text semantic annotator developed also by the NLM. MetaMap recognizes and disambiguates clinical terms in texts, associating to them its most likely UMLS CUI. MetaMap does not really exploit sentence context, but relies on a series of similarity metrics based to a large degree on morphological analysis to match entities to UMLS concepts. MetaMap is available as a (RESTFul) webservice ${ }^{9}$.

BabelFly. BabelFly [12] is a state-of-the-art semantic annotator and entity linker that assigns to each (generic) entity $e$ a BabelNet babelsynset $s$. Similarly to Lesk, it also relies on sentence context, but instead of bag-of-words similarity it exploits the graph structure of thesauri (induced by lexical relations of synonymy, hypernonymy meronymy, etc.) to maximize a measure of clique similarity. As a side effect, it also returns the DBpedia URI associated to $s$ as well as its lemmas in all the languages covered by the babelsynset. BabelFly is available as a (RESTFul) webservice ${ }^{10}$.

Tagme. TagMe [4] is an annotator that links words to Wikipedia pages (i.e., to Wikipedia page URLs). TagMe works, broadly speaking, by jointly maximizing the coherence of all the (generic) entities $e_{1}, \ldots, e_{k}$ spotted in a sentence $S$. By coherence is understood a measure of topical relatedness among such terms and Wikipedia entries. TagMe covers currently two languages, English an Italian, and is also available as a (RESTFul) web service ${ }^{11}$.

\subsection{Evaluation}

The SemRep corpus. We ran our experiments over the SemRep corpus [9], a small annotated clinical corpus consisting of 428 clin-

\footnotetext{
${ }^{8}$ http://linkedlifedata.com 9 https://metamap.nlm.nih.gov

${ }^{10}$ http://babelfly.org ${ }^{11}$ http://tagme.di.unipi.it
} 

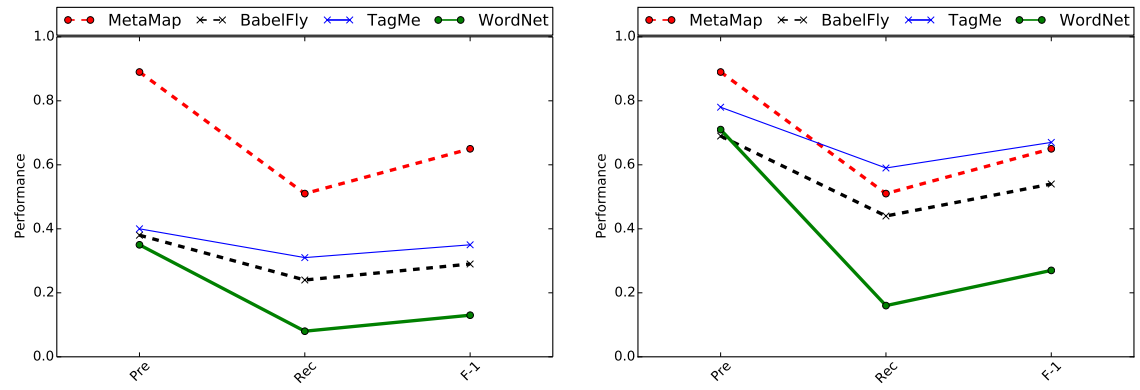

\begin{tabular}{l|l|l|l} 
Exp. & Test & Null hyp. & $p$-value \\
\hline \hline Unres. & Kruskal-Wallis & identical & 0.051 \\
Res. & Kruskal-Wallis & identical & 0.056 \\
& \multicolumn{3}{|l}{} \\
Test & Annotator & Null hyp. & $p$-value \\
\hline \hline$t$-test & BabelFly & identical & 0.015 \\
$t$-test & TagMe & identical & 0.008 \\
$t$-test & WordNet & identical & 0.151
\end{tabular}

Figure 2: Left: Annotation performance with unresolved DBpedia URIs. Middle: Annotation performance with resolved DBpedia URIs. Right, top: Statistical significance analysis of results; comparison of Pre, Rec and F1 among MetaMap, BabelFly, TagMe and WordNet baseline. Right, bottom: Statistical significance analysis of URI resolution; BabelFly and TagMe show a strongly significant improvement.

ical excerpts (MedLine/PubMed) and 13, 948 word tokens, roughly representative of the whole clinical domain. For each sentence, two noun phrases were annotated with their corresponding UMLS CUI by clinicians and domain experts, giving rise to a total of 856 UMLS-annotated clinical entities. Of these, 606 can be associated to a corresponding DBpedia URI. See Figure 3, left.

Annotation procedure. We ran the 4 annotators separately over the SemRep corpus. For each of the annotators (WordNet baseline, MetaMap, BabelFly, TagMe) we collected two kinds of information: (1) the corresponding entity sense annotations for the Gold corpus entities, together with their associated DBpedia URIs, whenever defined, viz., two per sentence; (2) for each sentence in the corpus, the senses of all the sentence's entities, their DBpedia URIs and their glosses ${ }^{12}$. See Figure 1, left, to get a glimpse as to how such annotations look like.

URI resolution. Different annotators may return senses that are only superficially different, and constitute only notational or lexical variants of the same synset or concept. In Figure 1, left, MetaMap returns http://dbpedia.org/resource/Parkinson_Disease, and TagMe returns http://dbpedia.org/resource/Parkinson's_Disease, two superficially different albeit identical URIs modulo identity. To understand how much impact this phenomenon has on annotator performance we additionally resolved such identities. Specifically, we tested for owl:sameAs and dbpedia:redirects relationships among the corresponding DBpedia URIs. On average, we discovered that one out of three of the URIs associated either to the corpus' CUIs or to the senses returned by the annotators is a variant (modulo ow 1 : sameAs and dbpedia:redirects) of a previously introduced DBpedia URI. See Figure 3, left.

Annotation performance. We evaluated MetaMap, BabelFly, TagMe and our Lesk-driven WordNet WSD baseline over the 606 DBpedia-annotated entities of the SemRep gold standard, and measured their precision (Pre), recall $($ Rec $)$ and F1-measure $(F 1)^{13}$, w.r.t. the unresolved and the resolved DBpedia URIs.

The results of the evaluation are summarized by Figure 1 . As expected, MetaMap achieved the highest precision, although at the expense of relatively low recall -over the clinical domain itself-: Pre $=0.89, \operatorname{Rec}=51, F 1=65$. Regarding the other annotators, again, as expected, our WordNet baseline performed uniformly worst, but TagMe surprisingly achieved better results than BabelFly, and even slightly surpassed the F1 score of MetaMap (0.67) when URIs where resolved (while achieving 0.78 preci-

\footnotetext{
12 BabelFly and TagMe were called with their default parameters. 13 Where: Pre $=\frac{\# \text { corrent senses }}{\text { \#returned senses }}, \operatorname{Rec}=\frac{\# \text { corrent senses }}{\# \text { corpus senses }}, F 1=\frac{2 \cdot \text { Pre } \cdot \text { Rec }}{\text { Pre }+ \text { Rec }}$.
}

sion $)^{14}$. Such differences were close to significant. Moreover, as the reader can see, while resolving URIs had little to no impact on MetaMap, it did improve significantly the results of all the other annotators ${ }^{15}$.

Gloss similarity performance. Given a clinical (generic) entity $e$ annotated with sense $s$ in the SemRep corpus, we measured the similarity of the corresponding sense $s^{\prime}$ returned by MetaMap, BabelFly, TagMe and WordNet by measuring how many times they were (1) WordNet related and (2) distributionally related ; we considered moreover two relatedness thresholds, a "strict" threshold $>0.2$ and a "loose" threshold $>0$. This gave rise to the following four similarity metrics. Respectively, WordNet similarity, WordNet loose similarity, distributional similarity and distributional loose similarity:

$$
\begin{aligned}
& \operatorname{syn}\left(s, s^{\prime}\right)=\frac{\sum\left\{\left(w, w^{\prime}\right) \in g(s) \times g\left(s^{\prime}\right) \mid w n_{>0.2}\left(w, w^{\prime}\right)\right\}}{|g(s)|+\left|g\left(s^{\prime}\right)\right|} \\
& \operatorname{syn}^{+}\left(s, s^{\prime}\right)=\frac{\sum\left\{\left(w, w^{\prime}\right) \in g(s) \times g\left(s^{\prime}\right) \mid w n_{>0}\left(w, w^{\prime}\right)\right\}}{|g(s)|+\left|g\left(s^{\prime}\right)\right|} \\
& \operatorname{dsyn}\left(s, s^{\prime}\right)=\frac{\sum\left\{\left(w, w^{\prime}\right) \in g(s) \times g\left(s^{\prime}\right) \mid d n_{>0.2}\left(w, w^{\prime}\right)\right\}}{|g(s)|+\left|g\left(s^{\prime}\right)\right|} \\
& \operatorname{dsyn}^{+}\left(s, s^{\prime}\right)=\frac{\sum\left\{\left(w, w^{\prime}\right) \in g(s) \times g\left(s^{\prime}\right) \mid d n_{>0}\left(w, w^{\prime}\right)\right\}}{|g(s)|+\left|g\left(s^{\prime}\right)\right|}
\end{aligned}
$$

where $g(s)$ denotes the bag-of-words derived from $s^{\prime}$ gloss. For distributional similarity, we used the standard Wikipedia-trained word2vec word space models. For WordNet and BabelFly we retrieved the corresponding WordNet and BabelNet glosses of the returned sense. For TagMe, we considered as "gloss" (sense definition) the DBpedia resource URI's abstract. Finally, for MetaMap and the SemRep corpus itself, we considered as "glosses" the MeSH (or Medline) abstract LinkedLifeData assigns CUIs.

As the reader can see in Figure 3 (middle and right), this analysis gave rise to only slight differences among the annotators. Such differences were not statistically significant.

Discussion. The annotation results show that, while MetaMap (and arguarbly, similar UMLS-annotators) does indeed beat general purpose entity linking systems on precision these perform reasonably well, providing in addition broad coverage, in particular when URIs are resolved. TagMe even slighly surpasses in F1-score MetaMap

14 BabelFly achieved 0.69 precision and a F1-score of 0.54 by comparison. ${ }^{15}$ We report only those tests that yielded the lowest $p$-values. 


\begin{tabular}{ll} 
\# of CUIs in corpus (total) & $=856$ \\
\hline \hline \# of corpus DBpedia URIs & $=606$ \\
\# of resolved corpus URIs & $=404$ \\
\hline \# of MetaMap DBpedia URIs & $=343$ \\
\# of resolved MetaMap URIs & $=242$ \\
\hline \# of BabelFly DBpedia URIs & $=432$ \\
\# of resolved BabelFly URIs & $=269$ \\
\hline \# of TagMe DBpedia URIs & $=469$ \\
\# of resolved TagMe URIs & $=320$ \\
\# of WordNet DBpedia URIs & $=182$ \\
\# of resolved WordNet URIs & $=97$
\end{tabular}

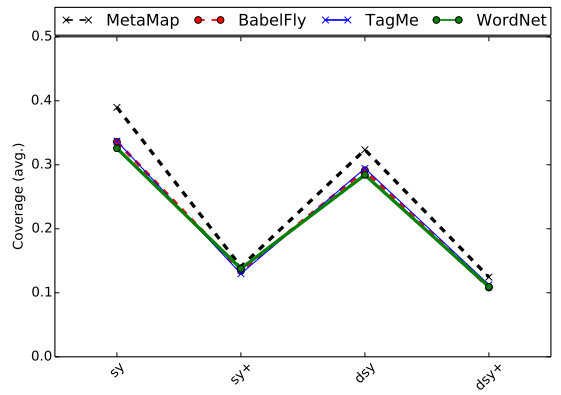

\begin{tabular}{l|c} 
Annotations & Avg. len. (per sent.) \\
\hline \hline Corpus sense glosses & 66.41 words \\
BabelFly sense glosses & 199.43 words \\
TagMe sense glosses & 325.51 words \\
MetaMap sense glosses & 191.76 words \\
WordNet sense glosses & 50.50 words
\end{tabular}

\begin{tabular}{l|l|l} 
Test & Null hyp. & $p$-value \\
\hline \hline Kruskal-Wallis & identical & 0.897
\end{tabular}

Figure 3: Left: Annotation statistics. On the first row, the total number of gold standard CUIs in SemRep. Next, the number of CUIs with an associated DBpedia URI (resp. a resolved URI). Finally, the number of annotations (resp. resolved) annotations returned by each annotator over the 606 DBpedia-linked entities of SemRep. Middle: Gloss similarity across annotators. Right, top: Average length of glosses (in content words), per sentence (the glosses were computed for all the noun phrases). Right, bottom: $A$ Kruskal-Wallis test showed no significant change between annotators.

(and all other annotators). We think that this is due to (1) their more advanced WSD algorithms, and, crucially, (2) the fact that they leverage encyclopedic knowledge that largely covers the clinical domain.

The results of the similarity evaluation show, on the other hand, that (3) sense glosses appear to be clinical domain related, but (4) are not sufficiently informative to distinguish the annotators. This may be due to the verbosity of the glosses, which (at a average, rate of 166.22 content words per sentence, all entities and annotators taken together), while broadly speaking remaining in the clinical domain, contain information not directly relevant for a term's sense definition.

\section{CONCLUSIONS}

We have carried out a cross-evaluation of domain specific (clinical) and cross- and multiple-domain semantic annotation systems over the SemRep clinical word sense disambiguation gold standard, to answer the question whether generic state-of-the-art systems can match in performance domain specific tools, while being able to semantically annotate both domain-specific and world-knowledge entities. In particular, we have compared two known entity linking systems, BabelFly and TagMe, that leverage on Wikipedia and DBpedia, plus a simple WordNet baseline, with the standard clinical semantic annotation and disambiguation system, MetaMap. Our results show that BabelFly and TagMe perform reasonably well over the clinical domain (their precision lying close to that of MetaMap), while achieving a much wider coverage (higher recall) than MetaMap. This is due to their leveraging on the large body of encyclopedic knowledge from Wikipedia, as the poor results of the baseline (restricted to WordNet) show.

\section{REFERENCES}

[1] A. R. Aronson and F.-M. Lang. And overview of MetaMap: Historical perspective and recent advances. Journal of the American Medical Informatics Association, 17(3):229-236, 2010.

[2] C. Bizer, J. Lehmann, G. Kobilarov, S. Auer, C. Becker, R. Cyganiak, and S. Hellmann. DBpedia - A crystallization point for the web of data. Journal of Web Semantics, 7(3):154-165, 2009.

[3] e. Christiane Fellbaum. WordNet: An Electronic Lexical Database. MIT Press, 1998.

[4] P. Ferragina and U. Scaiella. TAGME: on-the-fly annotation of short text fragments (by wikipedia entities). In
Proceedings of the 19th ACM International Conference on Information and Knowledge Management (CIKM 2010), 2010.

[5] G. Goth. Analyzing medical data. Communications of the ACM, 55(6):13-15, 2012.

[6] J. R. Hobbs. The generic information extraction system. In Proceedings of the Fifth Message Understanding Conference (MUC-5), 1993.

[7] E. Hovy, R. Navigli, and S. P. Ponzetto. Collaboratively built semi-structured content and artificial intelligence: The story so far. Artificial Intelligence, 194:2-27, 2013.

[8] I. Hulpus, C. Hayes, M. Karnstedt, and D. Greene. An eigenvalue-based measure for word-sense disambiguation. In Proceedings of the 25th International Florida Artificial Intelligence (FLAIRS 2012), 2012.

[9] H. Kilicoglu, G. Rosenblat, M. Fiszman, and T. C. Rindfleisch. Constructing a semantic predication gold standard from the biomedical literature. $B M C$ Bioinformatics, 12(486), 2011.

[10] M. Lesk. Automatic sense disambiguation using machine readable dictionaries: How to tell a pine cone from an ice cream cone. In Proceedings of the 5th Annual International Conference on Systems Documentation (SIGDOC 1986), 1986.

[11] V. Momtchev, D. Peychev, T. Primov, and G. Georgiev. Expanding the pathway and interaction knowledge in linked life data. Proceedings of 2009 International Semantic Web Challenge, 2009.

[12] A. Moro, A. Raganato, and R. Navigli. Entity linking meets word sense disambiguation: a unified approach. Transactions of the Association for Computational Linguistics, 2:231-244, 2014.

[13] R. Navigli and S. P. Ponzetto. BabelNet: Building a very large multilingual semantic network. In In Proceedings of the 48th Annuanl Meeting og the Association for Computational Linguistics (ACL 2010), 2010.

[14] A. Sajadi, E. E. Milios, V. Keselj, and J. C. M. Janssen. Domain-specific semantic relatedness from Wikipedia structure: A case study in biomedical text. In Proceedings of the 16th International Conference in Computational Linguistics and Intelligent Text Processing (CICLing 2015), 2015. 